\title{
Lower Handgrip Strength levels probably precede Insulin Resistance and Associated with Diabetes in Men not in Women
}

\section{Jia Zheng ( $D$ 1637643374@qq.com )}

Fourth Affiliated Hospital of China Medical University https://orcid.org/0000-0003-1382-5135

\section{Lu Zhang}

University of Pecs

Min Jiang

China Medical University

Original investigation

Keywords: handgrip strength, insulin resistance, diabetes

Posted Date: February 23rd, 2021

DOl: https://doi.org/10.21203/rs.3.rs-203394/v1

License: (c) (i) This work is licensed under a Creative Commons Attribution 4.0 International License.

Read Full License 


\section{Abstract}

\section{Background}

To explore the relationship between handgrip strength per weight (HGS/W) and insulin resistance and diabetes metabolism (DM), and whether the lower HGS levels precede IR in China elderly population.

\section{Methods}

Two linear regression models were employed to explore the association of whether baseline HGS/W predicted follow-up variation of triglyceride glucose index (TyG) or baseline TyG predicted follow-up variation of HGS/W. The logistic regression model was used to examine the relationship between baseline HGS/W and future DM.

\section{Results}

A total of 4,561 participants in China Health and Retirement Longitudinal Study were enrolled, of which $47.0 \%$ were men, and the mean age was 58.7 (standard deviation, 8.68) years. A lower level of baseline HGS/W was significantly correlate with a higher level of follow-up TyG $(\beta=-0.173, P=0.002)$. The baseline level of HGS/W was significantly negatively associated with incidence risk of DM (rate ratio = $0.375, P=0.004)$. However, in gender stratification, statistical association between HGS/W and TyG and DM was only in male.

\section{Conclusions}

Our results demonstrated that the HGS/W was inversely associated with IR and DM, and lower HGS/W levels preceded TyG levels in the elderly population. However, the effect was inconsistent between male and female, and the possible mechanism would need further clarified.

\section{Introduction}

Diabetes metabolism (DM) as one of the top cause of world death, is a chronic disease because of the resistant of insulin or the lack of enough insulin [1]. Over time, DM might develops into systemic diseases [2]. The patient number of DM is growing globally [3]. China ranked among the top five globally in morbidity and mortality of DM in 2017 [4], and these numbers are increasing [5]. However, more than half of DM (or pre-DM) patients are undiagnosed [6]. Besides, under the coronavirus 2019 (COVID-19) pandemic background, it was reported that diabetic patients have a higher chance of being a severe coronavirus patient and have a poor prognosis [7]. However, because of COVID-19, hospital resources are redistributed, and citizens must reduce social interactions, restricting medical testing and treatment for DM or pre-DM patients. Hence, it will reduce the future DM burden if there is a simple method for predicting diabetes, and patients or potential patients can self-test at home, and report to their family doctor online. 
Handgrip strength(HGS) test is a non-invasive, simple upper limb muscle function test [8]. HGS is becoming a predictor of heart disease, vascular disease, peripheral artery disease, nerve damage, and malnutrition [9]. Furthermore, some studies have explored the relationship between grip strength (GS) and DM at different ages and races $[10,11]$. Nevertheless, it is still unknown among the Chinese elderly. Therefore, this study aims to examine the correlation between HGS and DM in Chinese older people.

Muscle movement depends on glucose metabolism[12]. However, insulin resistance (IR) reduces the muscle's ability to process glucose and weakens muscle strength $[13,14]$. Besides, IR is one of the central mechanisms of DM progression [15]. Most DM patients, especially obese patients, are resistant to insulin. Hence, IR testing is used in early DM screening[16]. Therefore another goal of our research was to explore the temporal relationship between HGS and IR.

\section{Methods}

Study population

CHARLS is conducted by the National School of Development of Peking University and is a nationally representative survey. The national baseline survey was conducted from June 2011 to March 2012. A multistage, random cluster sampling process was performed in 450 villages/urban and 10,287 households with aged $\geq 45$ years as a representative sample was finally selected. Every two years, the same questionnaires as the baseline were periodically employed in these sample households. Besides, their blood samples were collected in 2011 and 2015. Peking University's ethical review committee (IRB 00001052-11014) had approved the study protocol. The other detailed information on the China Health and Retirement Longitudinal Study (CHARLS) website (http://charls.pku.edu.cn/en).

Our research was a post hoc analysis. Figure 1 describes the details of the sampling process. A total of 17,708 individuals were at baseline. We excluded subjects younger than 45 years $(n=1,099)$ or having diabetes, and cancer $(n=2,366)$ at baseline. Then, subjects were excluded if 2011 data were missing in ethnicity, education level,and gender $(n=1,413)$, or in height, weight, and hand grip strength $(2,989)$, or in SBP, DBP, or pulse $(n=131)$, or in HDL-C, LDL-C, TG, or FPG $(n=2,815)$. Thus, 6,895 participates attended the follow-up. Furthermore, subjects were excluded because of missing data in height, weight, or HGS $(\mathrm{n}=237)$, or in TG, or FPG ( $\mathrm{n}=2079)$ in 2015. Finally, 4,561 participates (2144 males and 2417 females) were included in this study.

\section{Exposures and Covariates}

Demographics (age, gender, education, and ethnicity), lifestyle factors (drinking and smoking status), and diseases (diabetes and cancer) were obtained through the structured home interview by CHARLS trained health staff. Besides, health behaviors information was collected from self-reported health questionnaire:1, frequency of alcohol consumption (never, once a month, and more than once a month); 2 , smoking status in the past year (never, former, and current smoker). 
What is more, CHARLS describes the blood pressure indexes (cholesterol indexes, FPG, biochemical blood indexes, and others) collection and measurement methods on their website (http://charls.pku.edu.cn/en). Then, we used an auto-analyser (Olympus AU640 Auto-Analyzer, Olympus Corp, Kobe, Japan) measured TG, LDL-C, and HDL-C and Omron ${ }^{\mathrm{TM}}$ HEM-7200 Monitor (Omron (Dalian) Co., LTD., Dalian, China) measured SBP, DBP and pulse in the sitting position in a five-minute rest interval, and Enzymatic colourimetric test method measured. Besides, the handgrip strength per weight (HGS/W) was calculated by HGS/W = handgrip strength $(\mathrm{Kg}) /$ weight $(\mathrm{Kg})$. And triglyceride glucose $(\mathrm{TyG})$ index was calculated through formula, which is TyG $=\ln$ [fasting $T G(\mathrm{mmol} / \mathrm{L})$ * FPG $(\mathrm{mmol} / \mathrm{L}) * 0.5 * 159.37$ ]

\section{Statistical analysis}

Percentiles, mean (standard deviation, SD), and median (interquartile range) were used to describe the central and discrete trends for categorical variables, normally distributed continuous variables, and nonnormally distributed continuous variables, respectively. Simultaneously, t-test, or Mann-Whitney U test, or Pearson's $\chi 2$-tests were used to compare the statistical significance between men and women.

Three regression models were built to examine associations between baseline HGS/W and future TyG, and $D M(X=H G S / W$ in 2011 and $Y=D M$ cases and $Y=T y G$ variation in 2015, respectively) and between baseline TyG and future HGS/W, and DM ( $X=$ TyG in 2011 and $Y=D M$ cases and $Y=H G S / W$ variation in 2015, respectively). Baseline HGS/W and TyG were respectively adjusted in Model 1. Besides, Model 2 was adjusted in baseline HGS/W, baseline TyG, sex, ethnicity, and age. Based on Model 2, Model 3 added the 2011-year education, current smoking, alcohol drinking, SBP, DBP, HDL-C, LDL-C variables. Then, the three models were constructed in groups of men and women, separately. Besides, Variance expansion factor (VIF) was examined for the multicollinearity among independent variables. VIF > 10 means variables significant in multicollinearity. Statistical significance was accepted when $P \leq 0.05$. All statistical analyses were performed in by IBM SPSS version 25.0.

\section{Results}

Of the 4,561 participants (mean age 58.7 (SD, 8.68) years ), 2144 were male and 2417 were femal. Population characteristics of baseline and follow-up are shown in Table 1. baseline SBP, DBP, HR, and FPG and futhure DM were no significant gender differences.

Table 2 showed the prospective association of baseline HGS/W with follow-up TyG and DM. From the the result of model 3 , we found that the level of HGS/W was inversely associated with the level of follow-up TyG $(\beta=-0.173, P=0.002)$. At the same time, we found that a higher level of HGS/W was related with a lower incidence risk of $\mathrm{DM}(\mathrm{RR}=0.375, P=0.004)$.

Table 3 showed the prospective correlation between baseline TyG and follow-up HGS/W and DM. We found that the level of TyG was positively correlated with the incidence risk of follow-up DM $(R R=1.712$, $P<0.001)$. However, the level of baseline TyG was not significantly related to the level of follow-up $\mathrm{HGS} / \mathrm{W}(\beta=-0.007, P=0.057)$. 
Table 4 showed the results of subgroup analysis by gender. For men, the level of HGS/W was inversely associated with the level of follow-up TyG $(\beta=-0.204, P=0.010)$ and a higher level of HGS/W was related to a lower incidence risk of $\mathrm{DM}(\mathrm{RR}=0.250, P=0.004)$. However, the level of baseline HGS/W variation were no related with the level of follow-up TyG variation $(\beta=-0.150, P=0.055)$ and the incidence risk of $\mathrm{DM}(\mathrm{RR}=0.577, P=0.270)$ for women.

\section{Discussion}

Until now, the conclusions of handgrip strength and diabetes is conflicting. And few research data concentrates on Chinese data. Therefore, we used the data of CHARLS from 2011 to 2015 to explore the relationship between GS and IR and DM in adults. The results of our study were 4-fold: (1) confirming that the baseline HGS/W was negatively related to future DM; (2) finding HGS/W was correlated with TyG; (3) verifying baseline HGS/W's levels preceded follow-up TyG'levels; (4) finding the effect of HGS/W on TyG and diabetes in men, not in women.

After controlling for confounding factors, we found that among Chinese older people, HGS/W was inversely associated with future DM. Previous studies support this finding. Mainous et al. showed that Americans over 45 years old with DM had a significant relationship with lower combined HGS [17]. Besides, in a South Korea nationwide survey, HGS, after adjusting for age, was negatively related to type 2 DM [11]. A 40-69 age group multi-ethnic UK study confirmed that a high HGS was associated with a low DM prevalence, and this correlation had ethnic differences $[10,18]$. Hence, our research complements supplementing the relationship between HGS and future DM in the Chinese older population.

In addition, some studies showed that there was no relationship between incident DM and HGS $[19,20]$ The DM cases in both articles were identified by self-report. Therefore, we thought the null findings could be caused by underestimating the number of DM cases. Another extensive multicentric research demonstrated that HGS was no associated with the incidence of T2DM [21]. A possible explanation for this study is the relatively short follow-up time and young age. As evidence, one study showed baseline HGS had a significant inverse relationship with the incidence of T2DM during 10-year follow-up time [22].

Although the potential mechanisms responsible for HGS's association with DM have not been fully understood, the following findings could explain the relationship between HGS and the incidence of DM. Defective glucose sensing at the beta cell and IR are two crucial pathophysiologic factors for abnormal glucose metabolism [23]. The levels of IR and the protein content of glucose transporter-4 can be increased by muscle strength training [24]. IR participates in the relation between diabetes and grip strength, which can be explained by the inactivation of insulin receptor, substrate-1 (IRS-1). Proinflammatory cytokines promote the phosphorylation of an IRS-1 by relevant protein kinases, such as JNK (c-JUN N-terminal kinase) and IKK $\beta$ (kappa-B kinase $\beta$ ) [25]. Then phosphorylated IRS-1 inhibits insulin sensitivity [25]. Besides, IL-6 can directly reduce IRS-1 activity [26]. Skeletal muscle is the principal place where insulin regulates glucose absorption [27]. Abnormal glucose and energy metabolisms cause the change of HGS. At the same time, our results have shown that the level of HGS/W at baseline was 
associated with the level of IR at follow-up, and the level of IR was positively correlated with the incident risk of diabetes. Hence, the IR mechanism may explain the negative correlations between HGS and DM.

Moreover, the temporal correlation results only showed that baseline HGS/W was related to future IR. The possible cause is that IR is an impaired response in the whole body, primarily in the liver, adipose tissue, and muscle [28]. Hence, early IR will not necessarily show up in muscles. However, if HGS levels decreased occurs early, IR will happen in the future. Hence, our result verifies that baseline HGS/W's level preceded the future IR level. Therefore, HGS can be a predictor of future IR and DM in older people [17].

Considering the gender dimorphism of HGS, we stratified gender. We only found that low GS levels were associated with IR and DM in the older male group. Similarly, a USA study in older adults study found a negative correlation between GS and FPG in male [29]. Moreover, white and black men had a stronger association between GS and DM than their women groups [18]. The same result was also be found in a 65-80 years old South Korean group[30]. Another study has demonstrated that GS was inversely related to type 2 diabetes incidence risk among 1632 men [31]. This study reported that the low GS could cause $27 \%$ population attributable fraction in men [31]. In addition, they found that the factor for incidence risk of type $2 \mathrm{DM}$ is reduced muscle strength in men [31]. The mechanism may be due to the chromosomes and sociocultural differences; males have relative more substantial muscle mass [32], higher inflammatory level [33] and lower insulin sensitivity [34] than females.

\section{Strength and limitation}

Two assumptions were crucial during our study. As our research was a strictly observational cohort study, the association between GS/W and DM may not be causal. Therefore, to minimize the potential bias of reverse causality, we excluded some individuals with the history of diabetes at baseline. Secondly, we can infer the temporal relationship between grip strength (GS/W) and incident DM as the prospective cohort design of CHARLS.

At the same time, our study also had some limitation. First, the crucial confounding factors, such as dietary patterns and physical activity (PA) were not considered in our study. Regular physical exercise could prevent age-related muscle strength loss [35] and increase insulin sensitivity [36]. On this basis, we are more confident that GS could be used as a useful predictor of the risk of DM. Another limitation is that the number of subjects are still relatively low, and we did not distinguish the Type 1 and Type 2 DM in our study. However, since Type 1 DM mostly occurs during adolescence, and the number of new occurrences in the elderly over 45 is very low, our study is still dominated by type $2 \mathrm{DM}$.

\section{Conclusions}

By combining all related research, HGS/W can be used as a simple, easy to measure and inexpensive indicator to predict IR and DM. HGS/W is more comfortable to be detected and monitored than biochemical indicators. Besides, HGS/W may help primary care physicians stratify the possible patients 
needing further blood test screening. Moreover, we need further studies to clarify the pathophysiological mechanisms underlying the relationship.

\section{Declarations}

Ethics approval and consent to participate: The procedures followed the ethical standards of the responsible committee of Peking University and the Chinese Center for Disease Control and Prevention, and written informed consents were obtained from all subjects or their proxies.

Consent for publication: Not applicable

Availability of data and materials: All the data can be found on the CHARLS website (http://charls.pku.edu.cn/en). The datasets analysed during our study are available from the corresponding author on reasonable request.

Competing interests: The authors declare that they have no competing interests.

Funding: This study received no external funding.

Authors' contributions: JZ contributed conception and design of the study; JZ organised the database; LZ and $\mathrm{MJ}$ reviewed the English language and grammar. JZ performed the statistical analysis, JZ and LZ wrote the first draft of the manuscript. All authors read and approved the final manuscript.

Acknowledgements: The authors are grateful to the CHARLS research team for providing the data. The authors thank Roland Ligetvári for providing medical editing support (proofreading, technical editing).

\section{References}

[1] World Health Organization. (2016) Global Report on Diabetes. World Health Organization.

[2] World Health Organization. Diabetes [Internet].

[3] Khan, M.A.B., Hashim, M.J., King, J.K., Govender, R.D., Mustafa, H. and Al Kaabi, J. (2020) Epidemiology of Type 2 Diabetes - Global Burden of Disease and Forecasted Trends. Journal of Epidemiology and Global Health, 10, 107-11. https://doi.org/10.2991/jegh.k.191028.001

[4] Lin, X., Xu, Y., Pan, X., Xu, J., Ding, Y., Sun, X. et al. (2020) Global, regional, and national burden and trend of diabetes in 195 countries and territories: an analysis from 1990 to 2025. Scientific Reports, Nature Publishing Group. 10, 14790. https://doi.org/10.1038/s41598-020-71908-9

[5] Li, Y., Teng, D., Shi, X., Qin, G., Qin, Y., Quan, H. et al. (2020) Prevalence of diabetes recorded in mainland China using 2018 diagnostic criteria from the American Diabetes Association: national cross sectional study. BMJ, British Medical Journal Publishing Group. 369, m997. https://doi.org/10.1136/bmj.m997 
[6] Yang, W., Lu, J., Weng, J., Jia, W., Ji, L., Xiao, J. et al. (2010) Prevalence of Diabetes among Men and Women in China. New England Journal of Medicine, Massachusetts Medical Society. 362, 1090101. https://doi.org/10.1056/NEJMoa0908292

[7] Selvin, E. and Juraschek, S.P. (2020) Diabetes Epidemiology in the COVID-19 Pandemic. Diabetes Care, American Diabetes Association. 43, 1690-4. https://doi.org/10.2337/dc20-1295

[8] Cronin, J., Lawton, T., Harris, N., Kilding, A. and McMaster, D.T. (2017) A Brief Review of Handgrip Strength and Sport Performance. Journal of Strength and Conditioning Research, 31, 3187-217. https://doi.org/10.1519/JSC.0000000000002149

[9] Rijk, J.M., Roos, P.R., Deckx, L., van den Akker, M. and Buntinx, F. (2016) Prognostic value of handgrip strength in people aged 60 years and older: A systematic review and meta-analysis. Geriatrics \& Gerontology International, 16, 5-20. https://doi.org/10.1111/ggi.12508

[10] van der Kooi, A.-L.L.F., Snijder, M.B., Peters, R.J.G. and van Valkengoed, I.G.M. (2015) The Association of Handgrip Strength and Type 2 Diabetes Mellitus in Six Ethnic Groups: An Analysis of the HELIUS Study. PloS One, 10, e0137739. https://doi.org/10.1371/journal.pone.0137739

[11] Lee, M.-R., Jung, S.M., Bang, H., Kim, H.S. and Kim, Y.B. (2018) Association between muscle strength and type 2 diabetes mellitus in adults in Korea: Data from the Korea national health and nutrition examination survey (KNHANES) VI. Medicine, 97, e10984.

https://doi.org/10.1097/MD.0000000000010984

[12] Abbatecola, A.M., Ferrucci, L., Ceda, G., Russo, C.R., Lauretani, F., Bandinelli, S. et al. (2005) Insulin Resistance and Muscle Strength in Older Persons. The Journals of Gerontology: Series A, 60, 1278-82. https://doi.org/10.1093/gerona/60.10.1278

[13] Shi, J., Fan, J., Su, Q. and Yang, Z. (2019) Cytokines and Abnormal Glucose and Lipid Metabolism. Frontiers in Endocrinology, Frontiers. 10. https://doi.org/10.3389/fendo.2019.00703

[14] Johnson, A.R., Milner, J.J. and Makowski, L. (2012) The inflammation highway: metabolism accelerates inflammatory traffic in obesity. Immunological Reviews, 249, 218-38. https://doi.org/10.1111/j.1600-065X.2012.01151.x

[15] DeFronzo, R.A. (2004) Pathogenesis of type 2 diabetes mellitus. Medical Clinics of North America, 88, 787-835. https://doi.org/10.1016/j.mcna.2004.04.013

[16] Tang, Q., Li, X., Song, P. and Xu, L. (2015) Optimal cut-off values for the homeostasis model assessment of insulin resistance (HOMA-IR) and pre-diabetes screening: Developments in research and prospects for the future. Drug Discoveries \& Therapeutics, International Research and Cooperation Association for Bio \& Socio-Sciences Advancement. 9, 380-5. https://doi.org/10.5582/ddt.2015.01207 
[17] Mainous, A.G., Tanner, R.J., Anton, S.D. and Jo, A. (2015) Grip Strength as a Marker of Hypertension and Diabetes in Healthy Weight Adults. American Journal of Preventive Medicine, 49, 8508. https://doi.org/10.1016/j.amepre.2015.05.025

[18] Ntuk, U.E., Celis-Morales, C.A., Mackay, D.F., Sattar, N., Pell, J.P. and Gill, J.M.R. (2017) Association between grip strength and diabetes prevalence in black, South-Asian, and white European ethnic groups: a cross-sectional analysis of 418656 participants in the UK Biobank study. Diabetic Medicine, 34, 11208. https://doi.org/10.1111/dme.13323

[19] Katzmarzyk, P.T., Craig, C.L. and Gauvin, L. (2007) Adiposity, physical fitness and incident diabetes: the physical activity longitudinal study. Diabetologia, 50, 538-44. https://doi.org/10.1007/s00125-006-0554-3

[20] Leong, D.P., Teo, K.K., Rangarajan, S., Lopez-Jaramillo, P., Avezum, A., Orlandini, A. et al. (2015) Prognostic value of grip strength: findings from the Prospective Urban Rural Epidemiology (PURE) study. The Lancet, 386, 266-73. https://doi.org/10.1016/S0140-6736(14)62000-6

[21] Marques-Vidal, P., Vollenweider, P., Waeber, G. and Jornayvaz, F.R. (2017) Grip strength is not associated with incident type 2 diabetes mellitus in healthy adults: The CoLaus study. Diabetes Research and Clinical Practice, Elsevier. 132, 144-8. https://doi.org/10.1016/j.diabres.2017.08.004

[22] Wander, P.L., Boyko, E.J., Leonetti, D.L., McNeely, M.J., Kahn, S.E. and Fujimoto, W.Y. (2011) Greater hand-grip strength predicts a lower risk of developing type 2 diabetes over 10 years in leaner Japanese Americans. Diabetes Research and Clinical Practice, 92, 261-4.

https://doi.org/10.1016/j.diabres.2011.01.007

[23] Ferrannini, E., Gastaldelli, A. and lozzo, P. (2011) Pathophysiology of prediabetes. The Medical Clinics of North America, 95, 327-39, vii-viii. https://doi.org/10.1016/j.mcna.2010.11.005

[24] Mizgier, M.L., Casas, M., Contreras-Ferrat, A., Llanos, P. and Galgani, J.E. (2014) Potential role of skeletal muscle glucose metabolism on the regulation of insulin secretion. Obesity Reviews: An Official Journal of the International Association for the Study of Obesity, 15, 587-97.

https://doi.org/10.1111/obr.12166

[25] Boura-Halfon, S. and Zick, Y. (2009) Phosphorylation of IRS proteins, insulin action, and insulin resistance. American Journal of Physiology-Endocrinology and Metabolism, American Physiological Society. 296, E581-91. https://doi.org/10.1152/ajpendo.90437.2008

[26] Dali-Youcef, N., Mecili, M., Ricci, R. and Andrès, E. (2013) Metabolic inflammation: Connecting obesity and insulin resistance. Annals of Medicine, Taylor \& Francis. 45, 242-53.

https://doi.org/10.3109/07853890.2012.705015 
[27] Nomura, T., Ikeda, Y., Nakao, S., Ito, K., Ishida, K., Suehiro, T. et al. (2007) Muscle strength is a marker of insulin resistance in patients with type 2 diabetes: a pilot study. Endocrine Journal, 54, 791-6. https://doi.org/10.1507/endocrj.k07-055

[28] Freeman, A.M. and Pennings, N. (2020) Insulin Resistance. StatPearls, StatPearls Publishing, Treasure Island (FL).

[29] Kalyani, R.R., Kim, C., Ferrucci, L., Laughlin, G.A., Kritz-Silverstein, D., Kong, S. et al. (2015) Sex differences in the association of fasting and postchallenge glucose levels with grip strength among older adults: the Rancho Bernardo Study. BMJ Open Diabetes Research \& Care, 3.

https://doi.org/10.1136/bmjdrc-2015-000086

[30] Chong, H., Choi, Y.E., Kong, J.Y., Park, J.H., Yoo, H.J., Byeon, J.H. et al. (2020) Association of Hand Grip Strength and Cardiometabolic Markers in Korean Adult Population: The Korea National Health and Nutrition Examination Survey 2015-2016. Korean Journal of Family Medicine, 41, 291-8. https://doi.org/10.4082/kjfm.18.0129

[31] Li, J.J., Wittert, G.A., Vincent, A., Atlantis, E., Shi, Z., Appleton, S.L. et al. (2016) Muscle grip strength predicts incident type 2 diabetes: Population-based cohort study. Metabolism, 65, 883-92. https://doi.org/10.1016/j.metabol.2016.03.011

[32] Pan, P.-J., Lin, C.-H., Yang, N.-P., Chen, H.-C., Tsao, H.-M., Chou, P. et al. (2020) Normative data and associated factors of hand grip strength among elderly individuals: The Yilan Study, Taiwan. Scientific Reports, 10. https://doi.org/10.1038/s41598-020-63713-1

[33] Visser, M., Pahor, M., Taaffe, D.R., Goodpaster, B.H., Simonsick, E.M., Newman, A.B. et al. (2002) Relationship of Interleukin- 6 and Tumor Necrosis Factor-a With Muscle Mass and Muscle Strength in Elderly Men and Women: The Health ABC Study. The Journals of Gerontology: Series A, 57, M326-32. https://doi.org/10.1093/gerona/57.5.M326

[34] Tramunt, B., Smati, S., Grandgeorge, N., Lenfant, F., Arnal, J.-F., Montagner, A. et al. (2020) Sex differences in metabolic regulation and diabetes susceptibility. Diabetologia, 63, 453-61. https://doi.org/10.1007/s00125-019-05040-3

[35] Al-Shreef, F.M., Al-Jiffri, O.H. and Abd El-Kader, S.M. (2015) Bone metabolism and hand grip strength response to aerobic versus resistance exercise training in non-insulin dependent diabetic patients. African Health Sciences, 15, 896-901. https://doi.org/10.4314/ahs.v15i3.25

[36] Krotkiewski, M., Lönnroth, P., Mandroukas, K., Wroblewski, Z., Rebuffé-Scrive, M., Holm, G. et al. (1985) The effects of physical training on insulin secretion and effectiveness and on glucose metabolism in obesity and type 2 (non-insulin-dependent) diabetes mellitus. Diabetologia, 28, 881-90. https://doi.org/10.1007/BF00703130 


\section{Tables}

Page 11/16 
Table 1 Baseline Characteristics of Study Participants by sex and abdominal obesity ( $\mathrm{n}=$ 4561)

\begin{tabular}{lcclc}
\hline Characteristic & Men $(\mathrm{n}=2145)$ & Women $(\mathrm{n}=$ & $P$ & Total \\
& & &
\end{tabular}

\section{Baseline (2011)}

Age (years)

$59.9(8.67)$

$57.8(8.59)$

$<0.001$

$58.7(8.68)$

Ethnicity, n (\%)

Han

Others

Education, n (\%)

Illiteracy
Primary school
Middle school
High school
Tertiary high school or
above
Current smoking, n (\%)
Drinking $\geq 1$ times/month,
SBP (mmHg)
DBP (mmHg)
Pulse (beats/minutes)
HDL-C (mg/dl)

LDL-C (mg/dl)
110.18(90.85- 132.60)

$664(31.0)$

632(29.4)

571(26.6)

94(4.4)

$1619(75.5)$

971(45.3)

129.1(20.07)

$75.7(12.50)$

71.7(10.76)

49.10(39.8259.92)

5.56(0.671)

1.31(0.852)

6.21(0.561)

$0.60(0.144)$

FPG (mmol/L)

TG (mmmol/L)

TyG

HGS/W
$184(8.6)$

$1435(59.4)<0.001$

464(19.2)

363(14.9)

128(5.4)

26(1.1)

183(7.6) <0.001

$184(7.6)<0.001$

129.2(21.48)

$0.945 \quad 129.1(20.82)$

75.2(11.74)

0.189

75.4(12.11)

72.1(9.60)

0.221

71.9(10.16)

$51.42(42.91-\quad<0.001 \quad 50.26(41.37-$ $61.08)$

$60.70)$

$118.30(97.04-\quad<0.001 \quad 114.43(93.94-$ 141.89)

136.86)

Follow-up (2015)

HGS/W

TyG

$0.54(0.145)$

$6.27(0.588)$

276(12.9)
$5.57(0.609)$

$1.44(0.843)$

$6.32(0.539)$

$0.44(0.128)$
0.459

$<0.001$

$<0.001$

$<0.001$
5.56(0.639)

$1.38(0.850)$

$6.27(0.553)$

$0.52(0.155)$
$\mathrm{DM}$

Abbreviations: GS/W, Grip Strength per body weight; TyG, triglyceride-glucose; SBP, systolic blood pressure; 
DBP, diastolic blood pressure; HDL-C: high-density lipoprotein cholesterol; LDL-C: low-density lipoprotein cholesterol; TG, Triglycerides; FPG, fasting blood glucose.

$\ln$ [fasting TG $(\mathrm{mmol} / \mathrm{L}) *$ FPG $(\mathrm{mmol} / \mathrm{L}) * 0.5 * 159.37]$.

HGS /W = grip strength $(\mathrm{Kg}) /$ weight $(\mathrm{Kg})$

Table 2 Prospective associations of baseline GS/W with follow-up TyG and DM

Follow-up TyG Incident DM

$\begin{array}{llll}\beta(95 \% \mathrm{CI}) & P & \mathrm{RR}(95 \% \mathrm{CI}) & P\end{array}$

\section{Modle 1}

GS/W

$-0.279(-0.372--0.185)$

$<0.001$

$0.333(0.188-0.590)$

$<0.001$

Modle 2

GS/W

$-0.246(-0.354--0.138)$

$<0.001$

$0.317(0.164-0.613)$

0.001

Modle 3

GS/W

$-0.173(-0.281--0.065)$

0.002

0.375(0.191-0.736)

0.004

Model 1: adjusted for baseline TyG.

Model 2: adjusted for factors in model 1 and baseline age, ethnicity and sex.

Model 3: adjusted for all variables in model 2 plus baseline education, current smoking, alcohol drinking, SBP, DBP, HDL-C, LDL-C.

Abbreviations: GS/W, Grip Strength per body weight; UA, uric acid; WC, waist circumference; TyG, triglyceride-glucose; SBP, systolic blood pressure; DBP, diastolic blood pressure; HDL-C: high-density lipoprotein cholesterol; LDL-C: low-density lipoprotein cholesterol. 
Table 3 Prospective associations of baseline TyG with follow-up GS/W and DM

\begin{tabular}{lcc} 
Follow-up GS/W & \multicolumn{2}{c}{ Incident DM } \\
$\beta$ (95\%CI) & $P$ & RR (95\%CI)
\end{tabular}

Modle 1

TyG $\quad-0.013(-0.019--0.006) \quad<0.001 \quad 1.781(0.527-2.076) \quad<0.001$

Modle 2

TyG

$-0.013(-0.019--0.006)$

$<0.001$

$1.835(1.572-2.142)$

$<0.001$

Modle 3

TyG

$-0.007(-0.014--0.000)$

0.057

$1.712(1.427-2.053)$

$<0.001$

Model 1: adjusted for baseline TyG.

Model 2: adjusted for factors in model 1 and baseline age, ethnicity and sex.

Model 3: adjusted for all variables in model 2 plus baseline education, current smoking, alcohol drinking, SBP, DBP, HDL-C, LDL-C.

Abbreviations: GS/W, Grip Strength per body weight; UA, uric acid; WC, waist circumference; TyG, triglyceride-glucose; SBP, systolic blood pressure; DBP, diastolic blood pressure; HDL-C: high-density lipoprotein cholesterol; LDL-C: low-density lipoprotein cholesterol. 
Table 4 Prospective associations of baseline GS/W with follow-up TyG and DM According to Sex
Follow-up TyG
Incident DM

$\begin{array}{llll}\beta(95 \% \mathrm{CI}) & P & \mathrm{RR}(95 \% \mathrm{CI}) & P\end{array}$

Men

Modle 1

$\mathrm{GS} / \mathrm{W}$

$-0.155(-0.305--0.005)$

0.043

$0.196(0.081-0.478)$

$<0.001$

Modle 2

GS/W

$-0.262(-0.418--0.107)$

0.001

0.255(0.101-0.646)

0.004

Modle 3

$\mathrm{GS} / \mathrm{W}$

$-0.204(-0.360--0.048)$

0.010

$0.250(0.097-0.64)$

0.004

Women

Modle 1

GS/W

$-0.224(-0.375--0.072)$

0.004

$0.314(0.122-0.808)$

0.016

Modle 2

GS/W

$-0.242(-0.395--0.089)$

0.002

0.391(0.150-1.018)

0.054

Modle 3

GS/W

$-0.150(-0.303-0.003)$

0.055

0.577(0.218-1.531)

0.270

Model 1: adjusted for baseline TyG.

Model 2: adjusted for factors in model 1 and baseline age, ethnicity and sex.

Model 3: adjusted for all variables in model 2 plus baseline education, current smoking, alcohol drinking, SBP, DBP, HDL-C, LDL-C.

Abbreviations: GS/W, Grip Strength per body weight; UA, uric acid; WC,waist circumference; TyG,

triglyceride-glucose; SBP, systolic blood pressure; DBP, diastolic blood pressure; BMI, body mass index; HDL-C: high-density lipoprotein cholesterol; LDL-C: low-density lipoprotein cholesterol.

\section{Figures}




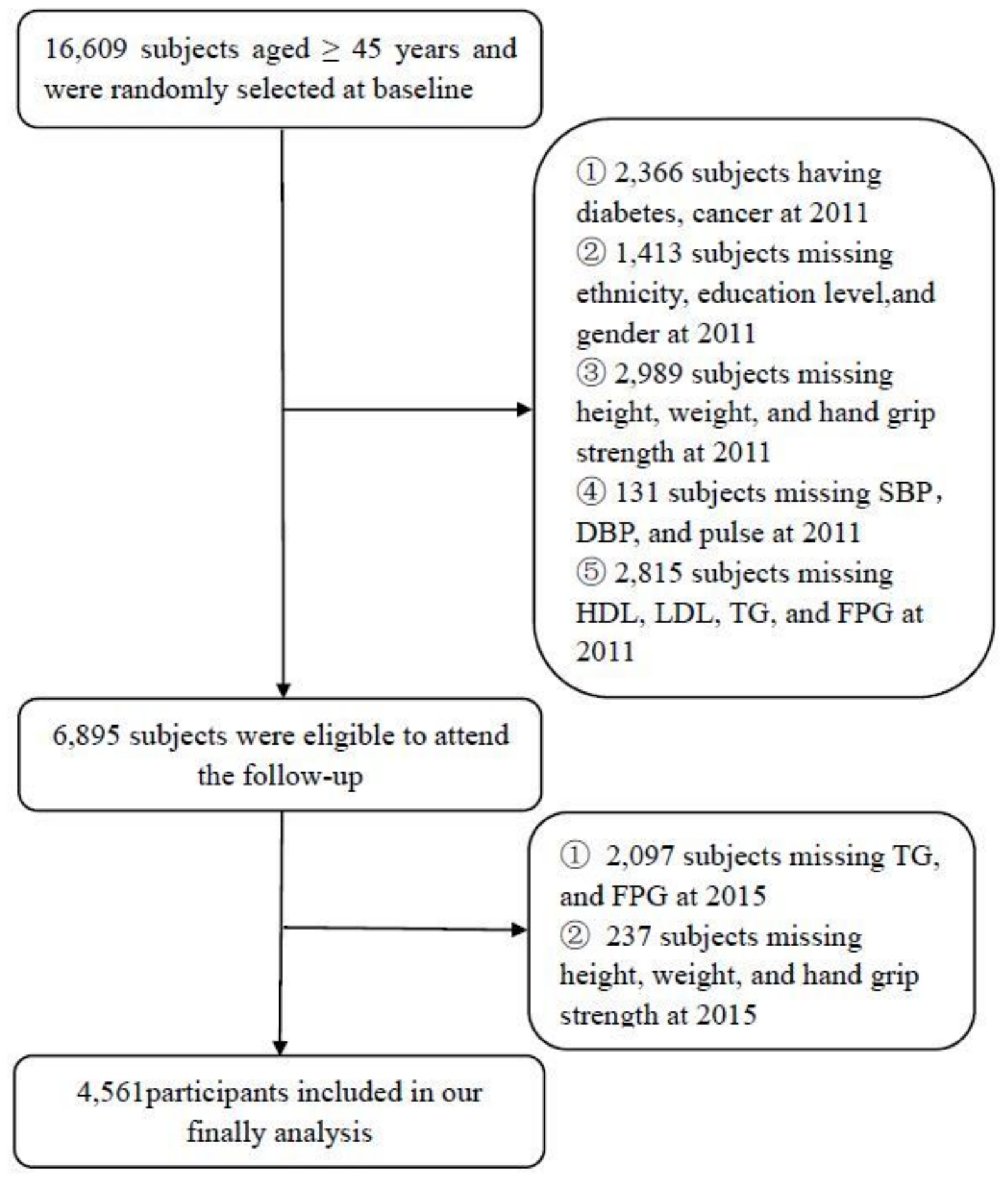

Figure 1

Flow chart for selection of study participants. 